\title{
Far-Field UHF Remotely Powered Front-End for Patient Monitoring with Wearable Antenna
}

\author{
Onur Kazanc ${ }^{1}$, José A. Rodríguez-Rodríguez ${ }^{2}$, Manuel Delgado-Restituto ${ }^{2}$, Franco Maloberti ${ }^{3}$, Catherine Dehollain ${ }^{1}$ \\ ${ }^{1}$ RFIC Group, Ecole Polytechnique Federale de Lausanne, Lausanne, Switzerland \\ ${ }^{2}$ Institute of Microelectronics of Seville (IMSE-CNM-CSIC) - University of Seville, Spain \\ Integrated Microsystems Laboratory, University of Pavia, Pavia, Italy
}

\begin{abstract}
The design of a wearable antenna for UHF band at $900 \mathrm{MHz}$ with an integrated front-end for a wireless sensor system targeting remote patient monitoring is presented. A wearable antenna for human body with $\mathbf{- 7 . 2} \mathrm{dB}$ gain is demonstrated using five layer torso model. The antenna can deliver $25 \mu \mathrm{W}$ of power to the rectifier at $\mathbf{4 . 6}$ meters distance from the base station emitting $P_{E I R P}=4 \mathrm{~W}$. Local power management circuitry consumes a total power of $4 \mu \mathrm{W}$ while delivering $10 \mu \mathrm{W}$ of power for the sensors of the wireless sensor system. The system designed with $0.18 \mu \mathrm{m}$ CMOS process demonstrates the performance of the frontend together with 3D electromagnetic simulation results of the wearable antenna.
\end{abstract}

\section{INTRODUCTION}

Nowadays, biomedical healthcare sector is demanding increasingly new systems which are pushing boundaries of remote patient monitoring. The ability of a wearable wireless sensor to monitor, record and response to certain sensing information are identified as important roles on this goal. A wearable wireless sensor consists of a passive transponder with a sensor circuitry that provides information about the biological constants and an antenna that collects the incoming RF signal. Normally, this type of device is controlled by a RF base station by means of a master-slave communication where the base station and the wireless sensor are represented as master and slave, respectively.

Far-field remotely powered sensors allow the use of multiple sensors on human body with a single base station therefore avoiding multiple near-field readers. This provides increased mobility for the patient which in result serves better healthcare choices thanks to the far-field wireless operation [1]. Two main challenges on the design of these systems can be identified as a) Reduced amount of received power by the antenna because of the gain degradation while operating next to lossy human tissue b) Stringent power consumption specifications. These challenges should be addressed to enable proper operation while maximizing the operation distance of the sensor from the base station.

This paper presents the design of an energy harvesting frontend and power management circuit for a wearable wireless sensor system. In Section II, the design methodology of the wearable antenna will be explained aiming improved efficiency. Next, the energy harvesting circuitry will be presented in detail in Section III. Finally, the simulation results will confirm the feasibility of the whole system which is designed with the proposed methodology.

\section{WeARABle Antenna DESIGN FOR UHF BAND}

Fig. 1 shows the proposed wearable antenna. It is a planar structure built over a thin and flexible substrate made of polymide and it uses copper for the printed routing lines. Additionally, a foam layer with dielectric constant close to the air is placed beneath the polymide substrate to reduce the losses caused by the human body [2]. The antenna consists of a resonant radiating body and an inductively coupled feed-loop with input terminals for the active circuitry [3]. It has been designed so as to approximately provide conjugate impedance matching with the energy harvester RF front-end, thus, avoiding the use of additional passive components. The antenna impedance, $Z_{A N T}=R_{A N T}+j X_{A N T}$, can be expressed as,

$$
R_{A N T}=\frac{(2 \pi f M)^{2}}{R_{R B}} \quad \text { and } \quad X_{A N T}=2 \pi f L_{\text {Loop }}
$$

where $M$ is the mutual inductance between the radiating body and the feed-loop of the antenna; $L_{\text {Loop }}$ is the inductance of the feed-loop; and $R_{R B}$ is the resistance of the radiating body [4]. Mutual inductance $M$ strongly depends on the separation between the radiating body and the feed-loop, whereas $L_{\text {Loop }}$ is dominated by the length of the feed-loop. It is seen in (1) that $R_{A N T}$ and $X_{A N T}$ can be set independent of each other therefore the antenna structure provides high flexibility for conjugate impedance matching. Special attention has been

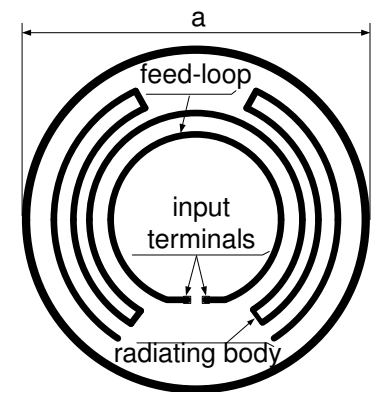

Fig. 1. Wearable antenna layout of the sensor system.

paid on the evaluation of the impact of the human body on the antenna performance. Fig. 2(a) shows the model employed in the $3 \mathrm{D}$ electromagnetic simulation, where the antenna is put over the proposed torso model. Fig.2(b) shows the stack of materials used on the antenna realization together with background materials that are representing simplified human torso model [5]. The figure depicts the thickness, dielectric constant and tangent loss of each material for $900 \mathrm{MHz}$ [6]. 


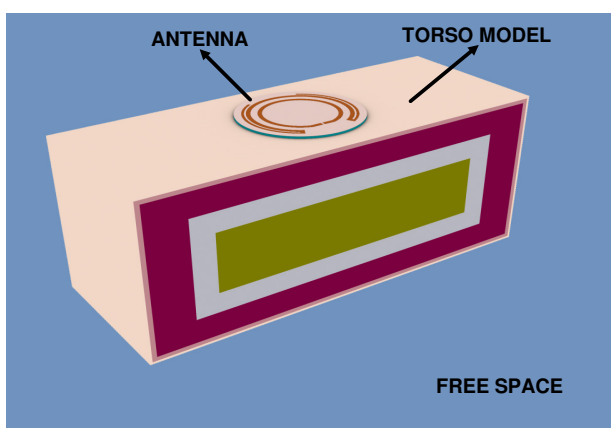

(a) 3D model of the antenna on simplified torso.

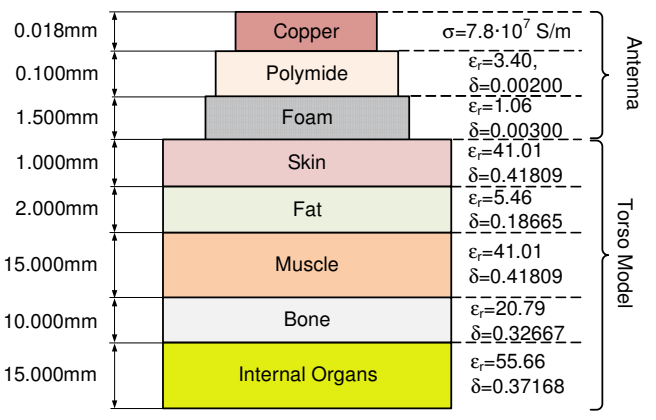

(b) Detailed stacked layers of 3D model.

Fig. 2. 3D model of the antenna and background material.

\section{INTEGRATED CIRCUITS}

\section{A. Rectifier and RF Frontend}

The RF Frontend of the sensor system shown in Fig.3 is composed of a rectifier, an envelope detector and and a backscattering modulator. The combination of three elements of the RF frontend together with bondwire parasitics enables accurate estimation of the input impedance of the rectifier therefore providing the necessary antenna impedance for maximum impedance matching for wireless power transfer.

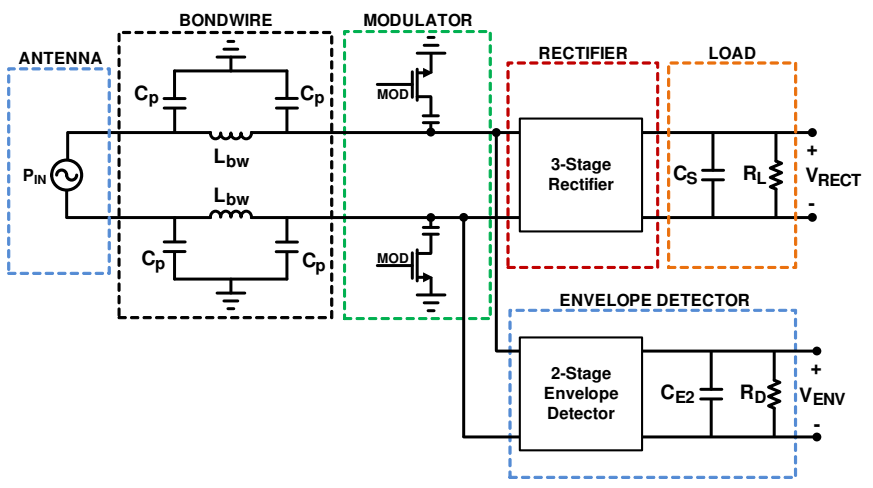

Fig. 3. RF frontend of the sensor system.

The three stage rectifier is composed of a cascaded highefficiency differential drive rectifier cell shown in Fig. 4. Self threshold voltage cancellation of the cell provides high power conversion efficiency with low input power although native MOS transistors are utilized [7]. The load of the rectifier is represented by $R_{L}$ and $C_{S} . R_{L}$ models the average DC load at the output of the rectifier for the power consumption of the baseband circuitry. $C_{S}$ acts as the supply capacitance for the sensor system by storing the charge provided by the rectifier.

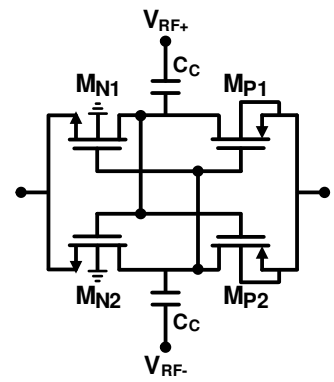

Fig. 4. Rectifier cell used in rectifier and envelope detector.

The envelope detector is the first stage of the demodulator circuit which has an identical topology to the rectifier as in Fig. 4 but differently sized to obtain a higher input impedance than the rectifier. As a result of this, most of the incident power is absorbed by the rectifier. The time constant of the load of the envelope detector is adjusted such that it can extract the data from ASK modulated carrier with the desired data rate.

The ASK backscattering modulator is implemented with an NMOS switch in series with a capacitor. The switch introduces the impedance mismatch between the antenna and rectifier in order to represent binary data while the series capacitor provides DC level shifting between the RF signal and the switch which prevents leakage of RF input power. Depending on the read range and local power management constraints, different modulation index $(m)$ values for the backscattering modulator can be adopted [8].

\section{B. Baseband power conditioning}

The voltage available at the output of the rectifier is used to supply the sensor system. The baseband power conditioning circuit depicted in Fig. 5 includes a power-on-reset (POR), a current reference embedded into bandgap voltage reference and a low-dropout regulator for providing stable supply voltage to the rest of the system.

1) V-I Reference and Power-on-Reset: The voltage and current $(V-I)$ reference of the sensor system is a self biased structure which is composed of a low-voltage bandgap reference [9] together with a current reference. In addition to those, a power-on-reset (POR) circuit is employed for the start-up sequence of the $V-I$ reference.

The reference current is imposed by the branch current of the bandgap reference through the drain of $\mathrm{P} 2$. The reference current can be written as,

$$
I_{D 2}=\frac{V_{T} \cdot \ln (N)}{R 1}+\frac{V_{B E 1}}{R 2}
$$

where $V_{T}$ is the thermal potential, $N$ is the multiplier of diode connected BJTs in D2, $V_{B E 1}$ is the forward diode voltage of $\mathrm{D} 1$. The resistors $\mathrm{R} 1$ and $\mathrm{R} 2$ determine the bias current for all the circuits as well as the power consumption of the bandgap reference. The branch current of the bandgap reference is mirrored to the current reference by the output voltage of the differential amplifier $V_{B P}$. The bias voltage for NMOS devices 


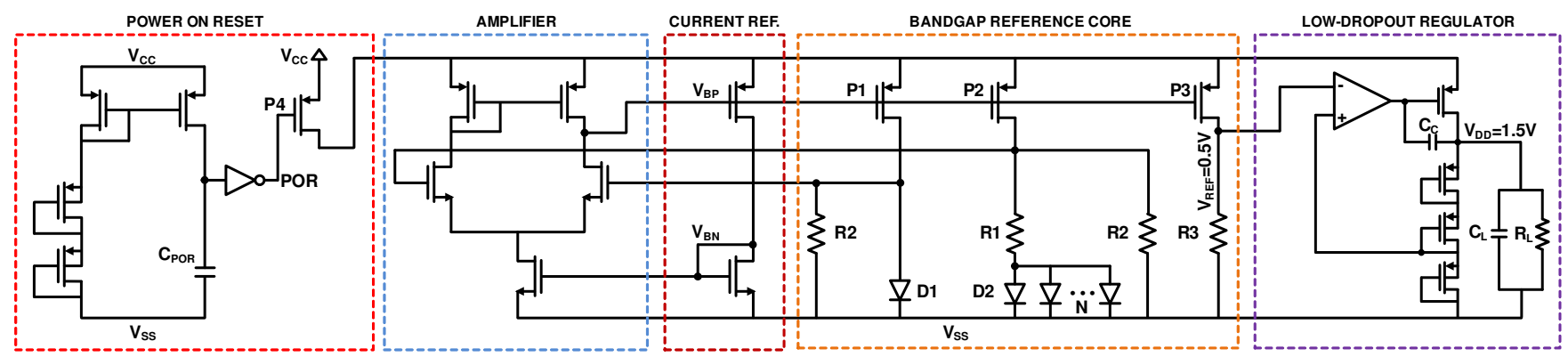

Fig. 5. Baseband power conditioning circuitry.

$V_{B N}$ is generated by the current reference block through the diode connected NMOS transistor. The single stage differential amplifier provides equal potentials across the diodes ensuring that two branches of the bandgap reference has identical currents $\left(I_{D 1}=I_{D 2}\right)$ for accurate operation of the bandgap reference. The reference voltage, which is generated by the current $I_{D 3}$ and resistor R3, is designed to be $0.5 \mathrm{~V}$ for the target sensor system.

The power-on-reset (POR) circuit provides a start-up pulse to $V$-I reference when the supply voltage $V_{R E C T}$ reaches to the desired threshold voltage of $V_{P O R}$. This ensures a correct operation point for the V-I reference in order to avoid zero output state. Additionally, by blocking the current flow through the circuits by $\mathrm{P} 4$ during initial charging of $C_{S}$, the startup time of the sensor system can be reduced. The circuit consumes a low quiescent current $(\mathrm{I}=60 \mathrm{nA})$ after the startup is initiated.

2) Low Dropout Regulator (LDO): The sensor system operates between different supply voltages as the voltage of the supply capacitor varies depending on the input power. Sensors, which require a stable supply voltage, has to be supplied with a voltage regulator in order to exhibit desired outputs. The conventional low-dropout voltage regulator provides $1.5 \mathrm{~V}$ supply voltage to the sensors. The regulator has a single ended differential amplifier that compares the MOS voltage divider to the reference voltage $V_{R E F}=0.5 \mathrm{~V}$ from the bandgap reference. The voltage divider is based on diode connected PMOS transistors. The source-bulk connected PMOS transistors avoid the body effect as $V_{S B}=0 \mathrm{~V}$ therefore ensuring equal division of the output voltage. The stability of the LDO is further improved by pole splitting through the internal compensation capacitor $C_{C}$.

\section{Simulation Results}

The necessary input power at the input of the RF frontend is determined by taking the power delivered to the load at the output of LDO, which is $10 \mu \mathrm{W}$, the power required for the baseband power conditioning circuit and efficiency of the rectifier $(\eta)$ into account. An input power of $25 \mu \mathrm{W}$ is calculated as minimum input power. The input impedance of the RF frontend is simulated as $Z_{I N, R F}=20-j 330 \Omega$ when $25 \mu \mathrm{W}$ is fed to the $\mathrm{RF}$ frontend. The wearable antenna is designed for the input impedance for conjugate matching resulting with $Z_{A N T}=20+j 330 \Omega$. By simulating with a fullwave 3D electromagnetic solver (CST Microwave Studio), the wearable antenna exhibits a gain of $-7.2 \mathrm{~dB}$ with a diameter of $46.2 \mathrm{~mm}$ and a total thickness of $1.6 \mathrm{~mm}$. The far-field radiation pattern of the antenna is depicted in Fig. 6. Next, the

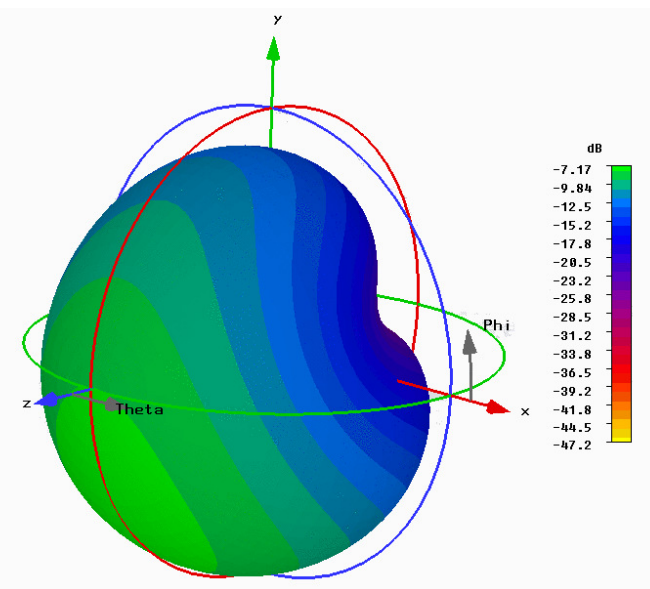

Fig. 6. Simulated far-field radiation pattern of the wearable antenna.

integrated frontend of the sensor system is simulated. Before the rectifier, $2.2 \mu \mathrm{W}$ of power is absorbed by the envelope detector therefore the total power delivered to the input of the rectifier is $22.8 \mu \mathrm{W}$. Providing an output power of $17.2 \mu \mathrm{W}$, the power efficiency of the rectifier is $75 \%$.

Assuming that the receiver has enough sensitivity for the backscattered signal, the read range is determined by the minimum input power, namely the sensitivity of the sensor system. The read range $r$ of a system with a sensitivity of $P_{M I N}=25 \mu \mathrm{W}$ can be calculated by [10],

$$
r=\frac{\lambda}{4 \pi} \sqrt{\frac{P_{E I R P} \cdot G_{R X} \cdot \tau}{P_{M I N}}}
$$

where $P_{E I R P}$ is the effective isotropic radiated power from the reader, $\lambda$ is the wavelength of the electromagnetic wave, $G_{R X}$ is the gain of the receiver antenna and $\tau$ is the power transmission coefficient between the receiving antenna and rectifier. By using simulated antenna gain of $-7.2 \mathrm{~dB}$ and $P_{\text {EIRP }}$ of four watts, the read range of the sensor system is calculated as 4.6 meters.

The start-up response of the integrated system driven by a power source of $25 \mu \mathrm{W}$ is plotted in Fig. 7. The storage capacitor $C_{S}$, which is $1.2 \mathrm{nF}$, reaches to start-up voltage $V_{P O R}=1.8 \mathrm{~V}$ and the POR signal goes from high to low then bias circuit is activated.

$C_{S}$ limits the instantaneous voltage drops in $V_{R E C T}$ during start-up sequence. Thus, it ensures proper operation of the bias 


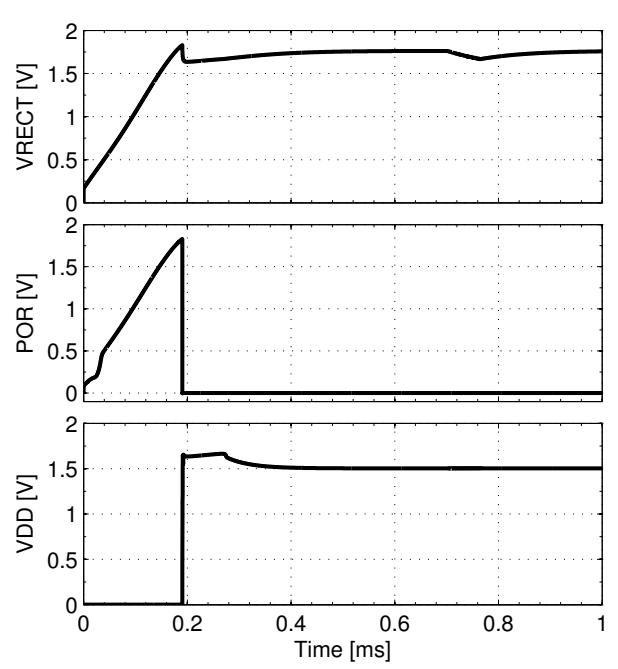

Fig. 7. Charging and start-up profile of the sensor system.

circuit during transients. The output of LDO reaches to $1.5 \mathrm{~V}$ and with a load of $225 \mathrm{k} \Omega$ at the output of the regulator, it can continuously deliver $10 \mu \mathrm{W}$ of power to the load. The dynamic performance of the system is depicted in Fig. 8. A modulated RF signal with a modulation index $m=1$, which can represent both transmit and receive links, includes 16-bits of zeros as a worst case scenario with a clock rate of $1 \mathrm{Mbit} / \mathrm{s}$. Each zero is encoded as 1110 in order to avoid excessive voltage drop on the storage capacitor during communication. The resulting voltage drop on the supply capacitor after 16-bit data transmission is $100 \mathrm{mV}$. The envelope detector extracts the received data from the modulated carrier with output level from $2.1 \mathrm{~V}$ to $0.4 \mathrm{~V}$. The baseband bias circuit together with the LDO consumes a total current of $2.3 \mu \mathrm{A}$ and a power of $4 \mu W\left(V_{C C}=1.75 \mathrm{~V}\right)$. In addition to that the baseband part of the envelope detector consumes $1 \mu \mathrm{W}$. The total power consumption of the system therefore becomes $15 \mu W$. Note that the difference between the rectifier output power (17.2 $\mu W)$ and the total power consumption of the system $(15 \mu W)$ is utilized to sustain the correct operation of the sensor system during communication phase. An ASK modulation depth $(m)$ different than one can mitigate this necessity.

\section{CONCLUSION}

The design of a remotely powered wearable sensor system for $900 \mathrm{MHz}$ is demonstrated. An isolation layer of foam is introduced between the torso and the antenna on the flexible PCB. The antenna designed with torso model exhibits a gain of $-7.2 \mathrm{~dB}$ with a total diameter of $46.2 \mathrm{~mm}$ and a thickness of $1.6 \mathrm{~mm}$. The system has a $75 \%$ of power conversion efficiency from RF to DC with an input power of $25 \mu \mathrm{W}$. Based on the power constraint of $10 \mu \mathrm{W}$ for the sensors, the sensor system can operate 4.6 meters distance from the base station while satisfying minimum input power threshold. The frontend with the envelope detector and modulator allows RX/TX communication with $1 \mathrm{MBit} / \mathrm{s}$ clock rate with a modulation index of $m=1$ while sustaining correct operation. The parameters of the sensor system are summarized in Table I.

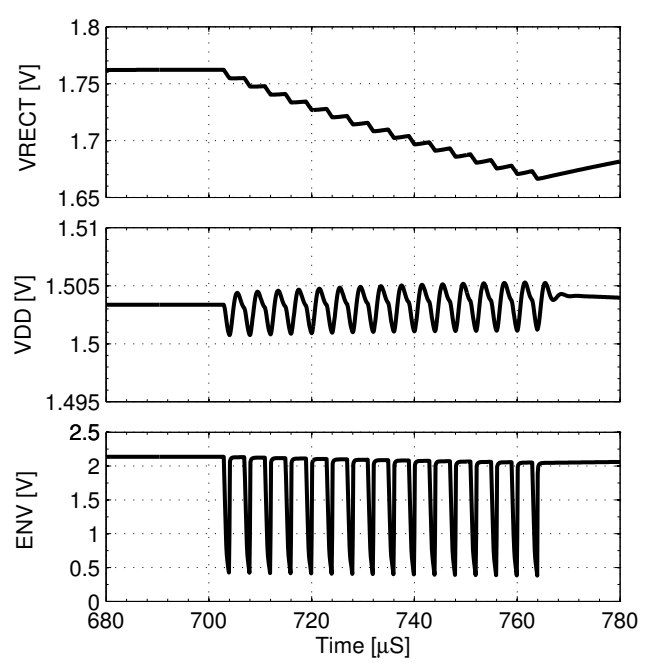

Fig. 8. Modulated carrier input response of the power management and the envelope detector.

TABLE I

SumMARY OF DESIGN PARAMETERS AND OUTPUTS

\begin{tabular}{cc}
\hline \hline Parameter & Value \\
\hline Operation Frequency & $900 \mathrm{MHz}$ \\
Available Input Power & $25 \mu \mathrm{W}$ \\
Operation Range $\left(P_{E I R P}=4 W\right)$ & 4.6 meters \\
Available Power for Sensors & $10 \mu \mathrm{W}$ \\
\hline
\end{tabular}

\section{ACKNOWLEDGMENT}

This work is funded by Nano-Tera initiative of Swiss National Funding (SNF) for project entitled "i-IRONIC" and the Spanish Ministry of Science \& Innovation under grant TEC2009-08447.

\section{REFERENCES}

[1] J. Penders et al, "Potential and challenges of body area networks for personal health", Annual International Conference of the IEEE Engineering in Medicine and Biology Society, pp.6569-6572, Sep. 2009

[2] W. Thompson, R. Cepeda, G. Hilton, M.A. Beach, S. Armour, "An Improved Antenna Mounting for Ultra-Wideband On-Body Communications and Channel Characterization", IEEE Transactions on Microwave Theory and Techniques, vol.59, no.4, pp.1102-1108, Apr. 2011

[3] A.H. Rida et. al., "Design, Development and Integration of Novel Antennas for Miniaturized UHF RFID Tags", IEEE Transactions on Antennas and Propagation, vol.57, no.11, pp.3450-3457, Nov. 2009

[4] H.W Son, C.S. Pyo, "Design of RFID Tag Antennas Using an Inductively Coupled Feed", Electronics Letters, vol.41, no.18, pp.994-996, Sep. 2005

[5] G. Marrocco, "RFID Antennas for the UHF Remote Monitoring of Human Subjects", IEEE Transactions on Antennas and Propagation, vol.55, no.6, pp.1862-1870, June 2007

[6] C. Gabriel, S. Gabriel and E. Corthout,"The Dielectric Properties of Biological Tissues: I. Literature Survey", Physics in Medicine and Biology, no. 41, pp.2231-2249, 1996

[7] K. Kotani, A. Sasaki, T. Ito, "High-Efficiency Differential-Drive CMOS Rectifier for UHF RFIDs", IEEE Journal of Solid-State Circuits, vol.44, no.11, pp.3011-3018, Nov 2009.

[8] U. Karthaus, M. Fischer, "Fully Integrated Passive UHF RFID Transponder IC with 16.7- $\mu$ W Minimum RF Input Power", IEEE Journal of Solid State Circuits, vol.38, no.10, pp.1602-1608, Oct. 2003

[9] H. Banba et. al., "A CMOS Bandgap Reference Circuit with Sub-1-V Operation", IEEE Journal of Solid State Circuits, vol.34, no.5, pp.670674, May 1999

[10] K.V.S. Rao, P.V. Nikitin, S.F. Lam, “Antenna design for UHF RFID Tags: A Review and a Practical Application", IEEE Transactions on Antennas and Propagation, vol.53, no.12, pp.3870-3876, Dec 2005 